\title{
Urban Developer: A model architecture for manageably building urban water cycle models spanning multiple scales
}

\author{
$\underline{\text { D. Snowdon }}^{\text {a }}$, M. J. Hardy ${ }^{\text {, }}$, J.M. Rahman ${ }^{\text {a }}$ \\ ${ }^{a}$ Commonwealth Science and Industrial Research Organisation (CSIRO) \\ ${ }^{b}$ BMT WBM, Australia \\ Email: dominic.snowdon@,csiro.au
}

\begin{abstract}
Urban Developer is a flexible and modular modelling environment for the simulation of urban water cycle services systems (potable water supply, stormwater, and wastewater). It enables the simulation of these systems at a range of spatial and temporal scales within a single framework and has been developed to facilitate an improved understanding of the potential of integrated water cycle management strategies.
\end{abstract}

Based on urbanCycle, the framework was designed to meet the objectives of scalability, ease of use, and complexity system management. The Urban Developer software application is built on the TIME framework and shares core functionality with the eWater Source IMS.

This paper discusses the four key software features of the Urban Developer framework that have been implemented to meet the stated design objectives. These are the implementation of: a dynamic node-link network editing canvas, a parameter 'styles' management system, support for nested or "layered" networks, and an adaptive time-stepping engine.

Urban Developer allows the linking together of water service components through the means of a node-link network. Each node in the network visually represents a system component, backed by a hydrological model of that component. Connection points corresponding to the water inputs and outputs are automatically added to each node. The components are connected by dragging a link from an output connector of a node to an input connector of another node, allowing the network to be rapidly built and easily visualised. With many instances of the same hydrological models, there is a large number of parameters to configure. This is managed by giving users the ability to create a new system component using an existing set of parameters as a template. This links the parameter set to the model, so changes to multiple identical models can be made simultaneously. To manage spatial complexity, a network can be nested in another network as a subnetwork node. This allows lower level details of the network to be hidden from high level views, and allows common network structures such as allotments to be built once and reused many times. The different temporal scale needs of the models are allowed for by a system of adaptive timestepping. When simulating periods of rainfall, short term response and thus fine simulation is required, while during dry periods only coarse simulation is required and thus the system clock is adjusted to a longer timestep. Processes are modeled coarsely where such a time scale is adequate, with inputs coming from finer scale models being automatically aggregated. While the timesteps used can be configured, the process is automatic, based on rainfall.

The Urban Developer Beta has enjoyed a high level of adoption and received much positive feedback. Additionally, developers on related eWater projects have commenced integrating some of the mentioned Urban Developer features into those other tools, showing some demand for them.

Keywords: Water Sensitive Urban Design (WSUD), urban water use, network visualisation, adaptive timestepping, nested network 


\section{MOTIVATION}

Integrated Urban Water Management (IUWM) is an emerging framework in which solutions to the provision of urban water services can be sought. It is based on the premise that, by removing the boundaries between the urban water cycle service systems and managing them as a whole, a more efficient use of resources can be achieved. It is anticipated that IUWM will promote new and innovative approaches to the provision of water cycle services that will incorporate greater levels of recycling and the reduction of wastes and better reflect the efficiencies of natural systems.

To date, no single model offers the ability to undertake the integrated modelling which is required to assess the performance of integrated urban water management options across the entire urban water cycle (Barry and Coombes, 2006). An extensive review of existing models further highlights an industry need for models that are capable of dealing with the urban water cycle in a holistic manner. (Breen et al., 2006).

By way of example, a holistic or "integrated" platform would enable users to carry out the assessment of multiple performance metrics across a system, to investigate the impacts of integrated management interventions that affect the response of multiple systems. For example, a rainwater tank utilised to meet domestic demand not only impacts the runoff characteristics of its catchments, but also the mains water supply system water balance as well.

Hardy (2009) identifies the major limitation of existing models to supported the simulation of integrated systems as: the spatial and temporal scale coverage of the urban water cycle service systems and the inability to informatively link system operations across scales; process representation, i.e. the need for the representation of all water cycle service systems; and limited flexibility with respect to network configuration and definition.

An integrated modelling tool therefore requires careful design to ensure that it is a useful tool, not only for modellers but also for planners, managers, and designers within the urban water sector. Attention must be given to managing the complexity of situations being represented, ensuring the operation of the model is scalable, and maintaining overall ease of use. This paper discusses the Urban Developer modelling framework and the key software feature implemented to address the major challenges of developing an integrated modelling platform.

\section{THE URBAN DEVELOPER MODEL}

Urban Developer is a next generation integrated urban water management software tool, which aims to meet the goals and challenges described above. It provides a framework for linking together urban hydrological models, allowing the simulation of each of the water services and the connections between them. This allows users to create an overall model of the situation they are investigating - or a new urban development they are planning - out of individual component models.

The User Interface (UI) of Urban Developer allows a user to design and execute networks of hydrological models. A network and related execution settings are contained within a scenario, and multiple scenarios are grouped into a project. The data associated with a project is saved to its individual file which can be shared with other users. Each scenario is designed and executed independently and only one project at a time can be open for use. Having multiple scenarios allows a user to explore multiple options and variations side by side. A scenario can be duplicated, allowing it to be used as the basis of other scenarios.

Each scenario has its own environmental configuration, which is comprised of rainfall, evaporation, and temperature. Given that the areas being modelled are typically small compared to the density of climate observations in urban areas, these inputs are considered to be consistent over all components within the scenario. There are multiple ways these environmental inputs can be obtained: by loading observed data, or by configuring design storm events.

\section{DISCUSSION}

Urban Developer is implemented using the TIME framework (Rahman et al., 2003) and shares a number of core components with the Source IMS (Argent et al., 2009, Welsh et al., 2011). It has been developed utilising $\mathrm{C} \#$ and is based on the research model urbanCycle (Hardy, 2009) which is written in FORTRAN. The household demand estimation component, known as the Behavioural End-use Stochastic Simulator 
(BESS) (Thyer et al., 2011) is in active development in FORTRAN so it is referenced by Urban Developer as a separate Dynamic Link Library (DLL).

Four primary Urban Developer features are Adaptive time-stepping, the Canvas, Styles, and Network Nesting. Adaptive time-stepping enables temporal resolution of the simulation to be heterogeneous in a controlled manner. The Canvas is the interface for users to create and visualise the network of hydrological models within a scenario. Styles assist in configuring the parameters of hydrological models, particularly in reusing common sets of parameters. Network Nesting is the key to managing the complexity of the different special scales, keeping them separate yet clearly connected. Together these features allow for complexity in a managed fashion, so Urban Developer can be flexible without being difficult to use.

\subsection{Adaptive Time-stepping}

In order to address the need to simulate model elements across a range of temporal scales, Urban Developer implements an adaptive time-stepping scheme through the underlying model engine. This scheme enables different periods of the simulation to have different temporal resolutions. Knowledge about how the models operate in certain environmental conditions is exploited to allow more efficient execution. There are three primary timesteps used by the adaptive time-stepping engine, each of which is a function of the rainfall state. During wet periods, short term response is required so a fine timestep is used. During dry periods, only a slow system clock is needed. In the transition period shortly after a wet period, no water is entering the stormwater system but it may still be flowing out, so an intermediary timestep is used to accurately model the residual flows.

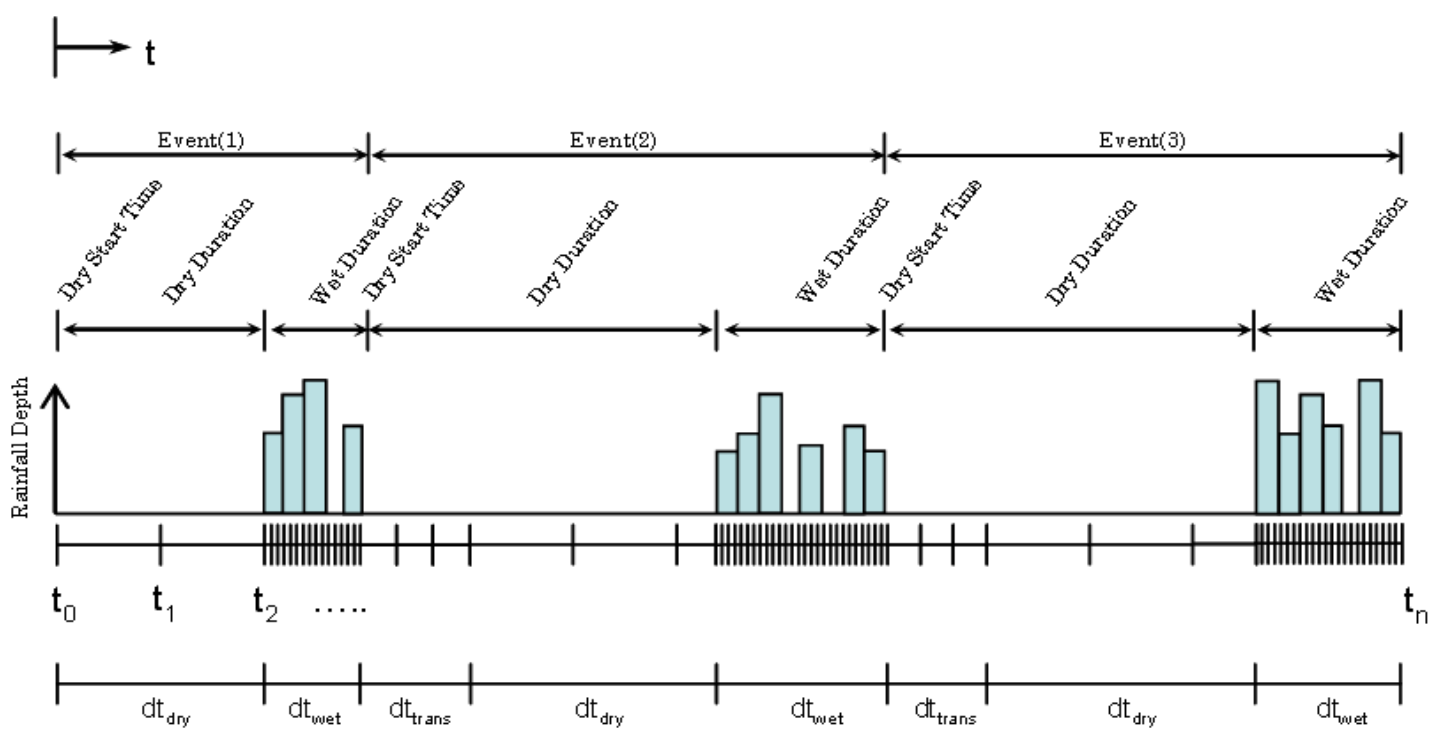

Figure 1. Time-steps varying throughout simulation according to rainfall Adapted from Hardy (2009)

Simulation of potable water demands always occurs at a single set timestep, independent of rainfall. As a result, not all components will follow the adaptive system clock. This results in links between models with timesteps of different lengths, which the engine handles via automatic aggregation of model inputs. This means that users don't need to consider different time-step lengths when constructing a network.

The major benefit of adaptive time-stepping is the reduction of simulation time. A constant timestep of two hours misses important details of rainfall events. A constant timestep of thirty seconds allows detailed modelling of storm response, but simulating dry periods at this level of precision is inefficient because there is no runoff. An adaptive system clock adaptive concentrates on the wet periods and skips quickly over the dry periods in which nothing occurs. Additionally, less memory is required to record the same results, and plotting the results is faster because fewer data-points are required to represent the data. 
In summary, the adaptive time-stepping scheme addresses the need to informatively link hydrological models across a range of temporal scales while at the same time providing simulation performance enhancements by reducing the overall model run times and memory requirements.

\subsection{Canvas}

The canvas is the interface for users to create, visualise, and edit a scenario's network, thus combining multiple hydrological models together. Within the canvas, models and the connections between them are represented as nodes (icons) and links (lines connecting the nodes) which form a network. The nodes on the canvas represent system components, such as pervious, impervious, and roof catchment areas, tanks, supplies, water use, outlets, and junctions. Each node is displayed as an icon and is simulated as an instance of the appropriate hydrological model. The Node Palette is a sidebar which presents all possible types of component that can be added to the canvas, which is done by dragging the palette icon onto the canvas. Once placed, a node can be dragged around the canvas, or it can be deleted if no longer needed. Most nodes have configurable parameters which control their behaviour, which can be edited in the Properties panel by clicking the model.

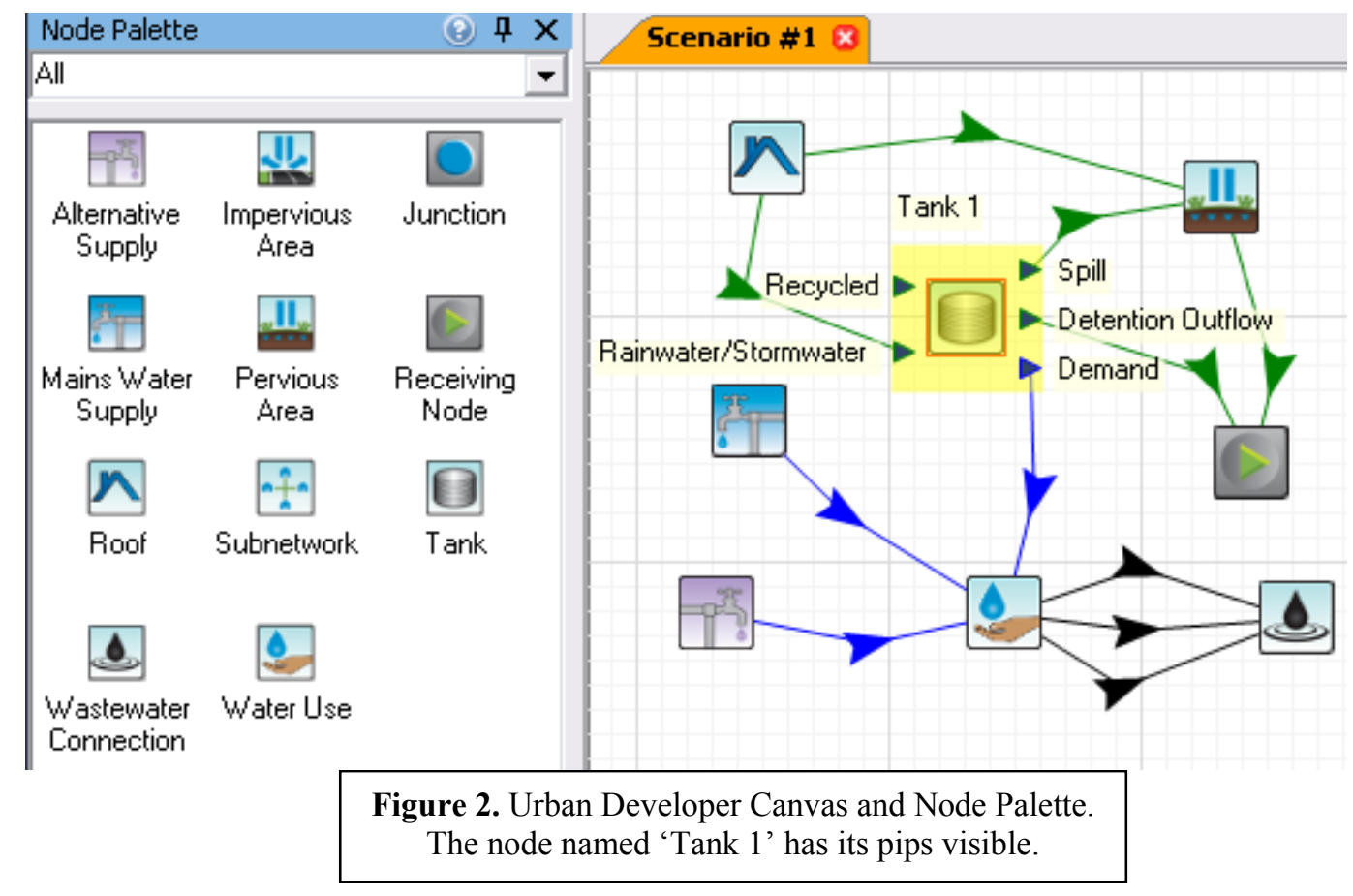

Models are connected via the flow of water from an output of one model to an input of another model. These are represented as connectors or 'pips' which are drawn on the sides of the node icons, with the inputs on the left and the outputs on the right. As a clutter-avoidance measure, node's pips are hidden when it is not in use. A link between two nodes - and thus between the underlying models - is made by clicking an output pip and dragging to an input pip. The canvas assists in the linking process by only showing compatible inputs. Also, the pips are colour-coded to denote what kind of water they represent, such as potable water, stormwater, and sewerage.

The links between nodes represent flows between models. They too are colour-coded, showing the water service which they represent. The user can toggle the visibility of all the links of each water service, in order to focus on particular services within the network. The direction of water flow through a link is shown by an arrow, which is helpful in understanding the network when the input/output pips are hidden. The arrow represents any link-routing model that exists between the connected nodes' models, and can also be dragged around the canvas to put a bend in the link, clicked to edit routing parameters, or deleted to remove the link.

As the purpose of multiple scenarios is the comparison of different design ideas, users can instantly view the canvas of any scenario they want to see. To assist in navigating large networks, standard pan and zoom functions are available. 
In summary, the canvas provides a clear interface to place and connect a variety of hydrological models, and to visualise and edit the network structure addressing the need for a flexible modelling environment to assist in the management of system complexity.

\subsection{Styles}

Urban Developer implements and extends the concept of styles developed in the urbanCycle modelling framework (Hardy, 2009). A Style is a named set of parameters for a specific type of hydrological model. A Style is similar to the text styles in a word processor: once a style has been defined it can be applied to multiple model instances, or it can be modified to affect all instances to which is has been applied. This allows multiple instances of the same type of component within an Urban Developer project to share the same parameters. Also components can be created using certain predefined sets of parameters. For instance, tanks may only be available in certain sizes, or a subdivision may only have two allowable sizes of roof.

The Styles system treats model parameters as a single unit that can be edited, switched, or copied separately from the network structure. With styles, the parameters of a node can be copied over to another node without needing to delete and recreate the target, which would require remaking the links between it and other nodes. Because styles are shared between all scenarios within an Urban Developer project, nodes in different scenarios can have the same styles. If multiples scenarios are required to contain some identical components, using styles ensures that changes cannot be made to model parameters in one scenario without updating all the others. Because styles are named, they make it easy to see what parameters a node has at a glance. This makes constructing and understanding a network much easier.

When a node is initially added to the canvas, it will have default parameters. It is in an unstyled state: it is not associated with a named style. Editing an unstyled node or link will only affect that single instance, unless the user makes a node styled. Dragging a node to the Style Manager will make a new style from the current parameters of the node, leaving the node in place as a styled node. Because the node is now styled, the name of the style will be visible below the node on the canvas. Now when the node is clicked, its style's parameters are visible in the Properties Panel and can be edited in the same way as a node's parameters.

The Style Manager is a sidebar, similar to the Node Palette. It presents a list of icons that can be dragged to the canvas to create new nodes, but they are styled nodes which share a style's parameters, rather than unstyled nodes with default parameters as the Node Palette creates. This method of creating a styled node makes a new node from an existing style. Clicking a style in the Style Manager also shows the style's parameters in the Properties Panel where it can be edited, and selects all nodes on the canvas that share the style. Styles remain in the Style Manger even if not used by any nodes, but can be deleted explicitly.

There are also methods of applying a pre-existing style to a pre-existing node. When viewing a styled node in the Properties Panel, a list of all styles appropriate to the node's hydrological model is available. This allows the user to simply select a different style from the list, immediately changing the model's parameters. The other method of applying a style to a styled or unstyled node is to drag a style from the Style Manager onto any compatible node on the canvas. Using these methods, the parameters of network components can be very rapidly changed between many different known groups of settings.

To assist in making styles easy to use, the various drag-drop operations follow a simple convention. All drag-drop operations for creating or moving nodes are done with the left mouse button. All operations for creating or setting styles are done with the right mouse button. Also for consistency, almost everything that can be done to and with nodes can also be done to and with the arrows on links. The exception is that link styles can't be used to create new link: they can only be applied to existing links.

Styles assist in managing the complexity of system models, however used in conjunction with nested networks, discussed in the following section, they aid in the support of spatial scale integration by facilitating the exploitation of hydrologically similar units that occur at a range of spatial scales within an urban catchment.

\subsection{Network Nesting}

Network Nesting is the primary feature aimed at managing the complexity inherent in the multi-level networks of the integrated urban water systems. As an example of the different layers: low-level components are connected to one another to represent a single allotment, multiple allotments form a cluster 
of houses, and these clusters together form a subdivision. If every connection between every component of every allotment in all the clusters in a large subdivision was visible at one time, the resulting network would be too complex to easily differentiate and navigate. This problem is addressed by nesting networks within 'subnetwork' nodes.

A subnetwork node acts like other nodes in its containing network, with input and output pips that enable users to connect it to other nodes in the network. However, instead of containing a hydrological model, a subnetwork node contains an entire network - a subnetwork within it. Each subnetwork is viewed and edited on a separate canvas. The simulation of subnetwork cannot be executed independently, rather only as a component of the parent network to which they belong and with which they interact.

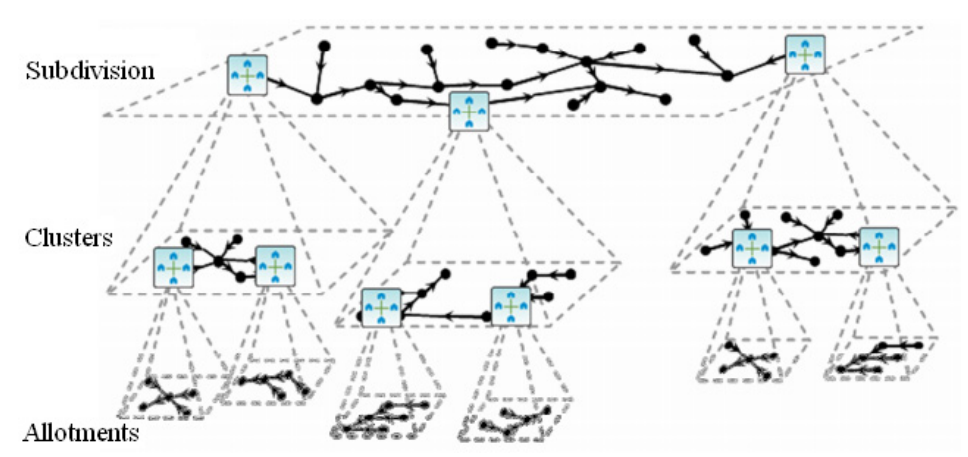

Figure 3. Multi-level network nesting using Subnetwork Nodes Adapted from Hardy (2009)

The main difference between a

subnetwork and a normal network is that a subnetwork contains special nodes which act as connections between the nodes inside the network and the input and output pips of the subnetwork node. These nodes are called 'supernet' connection nodes, indicating that they connect with the external parent network. The supernet connection nodes are created automatically within the subnetwork and cannot be deleted. Water that flows to an input of a subnetwork node is transferred to the corresponding supernet input connection node and then flows to whichever model is linked downstream, inside the subnetwork. Conversely, water flowing into a supernet output connection node is transferred out to the corresponding subnetwork node output, and then flows to the model downstream of the subnetwork node. In this manner, water flows into, through, and out of the subnetworks contained in subnetwork nodes.

Subnetworks are useful for keeping the layers of the network separate. On the top layer, just the connections between the various clusters in the subdivision are shown. This gives a high level view of the network structure and can be used to answer questions about aggregate and total flows. The mid-level view shows the makeup of a single cluster of houses, independent of other clusters and the details of how the clusters are connected or what the allotments contain. The fine details of the components within an allotment are visible on the lowest level, so they don't clutter up the higher levels. Subnetworks have an additional role in supporting reuse.

Subnetwork nodes can be styled the same way as normal nodes. The style of a subnetwork node is not a set of parameters: it is in fact the subnetwork. This means that two subnetwork nodes sharing a style have the same internal subnetwork, allowing users to create and reuse allotment and cluster styles. Rapid creation of networks is now possible because common low-level and mid-level network structures only need to be built once. Also, because the styles of nodes can be swiftly changed, the contents of an allotment or an entire cluster can be switched with a single drag-drop operation, making subnetworks a very powerful tool.

In short, Urban Developer's nested network structure helps users to model three separate layers of detail in an urban water system, while facilitating creation of meaningful connections between the different scales.

\section{OUTCOMES}

A beta version of Urban Developer was released in late February 2011. The beta was downloaded and used by over 170 people and received a great deal of positive feedback. The software tool was commercially launched as an eWater product on 5 July 2011, and the response to date has also been positive.

The adopted and developed modelling framework set out to address the key challenges identified for an integrate water cycle modelling framework. Its development drew on the existing research model 
urbanCycle (Hardy, 2009) for key strategies to address these issues but sought to implement them in a modern programming paradigm in order to deliver a commercially robust modelling framework to the industry.

While the numerical validity of Urban Developer's results has not be discussed in this paper, significant testing has been carried out to ensure that model behaviour is robust, and Urban Developer has been validated against other modelling packages where possible. As the product is quite new, it has to date had limited application by third party end users. These applications are commencing however, including - as an example - an application to Caloundra South in Queensland, by the consultancy WBM BMT. In that project Urban Developer was used to "quantify lot scale water cycle processes and to assess the impacts of rainwater capture and reuse, demand management and water recycling initiatives in achieving the potable water reduction/wastewater discharge requirements," using 10 years of local climactic and meteorological records (Bushell, 2011).

While Urban Developer has been released, further features remain to be added. Support for plugins will increase flexibility and capability. The simulation engine includes support for water quality analysis, but this is yet to be included in all the models and is hidden from the user interface. A final planned feature is upscaling, which is the linking of the outputs of an Urban Developer model into nodes in a regional scale model in another application. This allows the aggregate results of an urban development simulation to be used to drive a model operating at a larger scale, thus extending the tools applicability.

Further information on Urban Developer can be obtained from the eWater website http://www.ewater.com.au/uploads/files/Urban_Developer_Supporting_Papers.pdf

\section{REFERENCES}

Argent, R.M., Perraud, J.M., Rahman, J.M., Grayson, R.B., and Podger, G.M. (2009). A new approach to water quality modelling and environmental decision support systems, Environmental Modelling and Software, 24(7):809-818.

Barry, M., \& Coombes, P. J. (2006). 'The application of integrated water cycle management: an essential systems perspective.' Paper presented at the Integrated Urban Water Management: Challenges and Developments Workshop, held in conjunction with the 7th International Conference on Urban Drainage and 4th International Conference on Water Sensitive Design, Melbourne.

Breen, P., Coleman, J., Deletic, A., Duncan, H., Fletcher, T., Gerhart, T., et al. (2006). 'Integrated Urban Water Management Modelling Review Stage 2 Report.' eWater CRC Technical Report, Monash University, Victoria, Australia.

Bushell, S. (2011). Caloundra South and flood risk management. Council Leader, June 2011:42. ( http://www.ewater.com.au/uploads/files/Caloundra\%20Downs\%20short\%20for\%20Council\%20Lea der\%20magazine.pdf)

Hardy, M.J., McArthur, J. and Hardy M. (2011). Urban Developer Technical Overview. http://www.ewater.com.au/uploads/files/Urban_Developer_Technical_Overview.pdf : 2. Accessed $13 / 07 / 2011$

Hardy, M.J. (2009). 'Integrated Urban Water Management and the urbanCycle Modelling Framework.' PhD Thesis, University of Newcastle.

Thyer, M., Micevski, T., Kuczera, G., and Coombes, P. (2011). 'A Behavioural Approach to Stochastic End Use Modelling.' Paper presented at Oz Water, 9-11 May 2011, Adelaide.

Rahman, J.M., Seaton, S.P., Perraud, J.-M., Hotham, H., Verrelli, D.I., and Coleman, J.R. (2003). It's TIME for a new environmental modelling framework. Proceedings of MODSIM 2003,4: 1727-1732.

Welsh, W. D., Vaze, J., Dutta, D., Rassam, D., Rahman, J. M., Jolly I. D., Wallbrink P., Podger, G.M., Bethune, M. Hardy, M.J., Teng, J. And Lerat, J. (Under Review) An integrated Modelling Framework for Regulated River Systems. Environmental Modelling and Software. 